\title{
ANÁLISE DAS RELAÇÕES INTERPESSOAIS NA ÁREA DE PESQUISA E DESENVOLVIMENTO DE UMA INDÚSTRIA FAMILIAR
}

\section{1-Dusan Schreiber*}

Doutor em Administração pelo Programa de Pós-graduação em Administração da Universidade Federal do Rio Grande do Sul (PPGA/UFRGS), Brasil.

Professor do Mestrado Profissional em Indústria Criativa da Universidade Feevale, Novo Hamburgo/RS, Brasil. dusan@feevale.br

http://lattes.cnpq.br/5088882022022801

2- Vânia Gisele Bessi

Doutora em Administração pelo Programa de Pós-graduação em Administração da Universidade Federal do Rio Grande do Sul (PPGA/UFRGS), Brasil.

Professora do Mestrado Profissional em Indústria Criativa da Universidade Feevale, Novo Hamburgo/RS, Brasil vania@feevale.br

http://lattes.cnpq.br/5576556337944768

\author{
Diego Maganhotto Coraiola - Editor Geral \\ Editor responsável pela submissão: \\ Diego Maganhotto Coraiola. \\ Artigo analisado via processo de revisão duplo cego (Double-blind). \\ Recebido em: 26/05/2015 \\ Aprovado em: 13/05/2015 \\ Última Alteração: 21/06/2016
}




\title{
ANÁLISE DAS RELAÇÕES INTERPESSOAIS NA ÁREA DE PESQUISA E DESENVOLVIMENTO
} DE UMA INDÚSTRIA FAMILIAR

\section{RESUMO}

O universo organizacional configura-se como um recorte do mundo social, constituindo um ambiente complexo e multifacetado de relações sociais. Locus de realização e de identificação profissional e pessoal dos indivíduos que interagem, pode ser interpretado como campo de embate em defesa de interesses individuais e grupais, visando consolidar suas posições e acesso a recursos. $O$ artigo, elaborado com base em resultados de estudo de caso único e de análise de narrativas, teve como objetivo desenvolver análise compreensiva das relações interpessoais na área de P\&D de uma organização industrial familiar, da região sul do país. A análise foi realizada à luz de vertentes teóricas que versam sobre cultura organizacional, em virtude de sua relevância para o programa de gestão da inovação instituído pela organização. O teor das narrativas, elaboradas a partir de entrevistas em profundidade com onze técnicos facultou evidenciar a influência das relações interpessoais nesta área, sobre os critérios que norteiam as decisões no tocante à alocação de recursos e definição da forma de execução das atividades de pesquisa. Foi possível identificar aspectos da construção de relações interpessoais, bem como a manifestação de comportamentos com base na interpretação simbólica, que podem contribuir para a reflexão sobre as formas de gestão.

\section{Palavras-Chave}

Relações sociais, Relações interpessoais, Cultura organizacional, Empresa familiar.

\section{ANALYSIS OF INTERPERSONAL RELATIONS IN RESEARCH AND DEVELOPMENT OF A FAMILY INDUSTRY}

\begin{abstract}
The organizational universe represents society, in all details, as a complex and multifaceted social relations environment. As a locus of professional and personal identification of individuals who interact, it can be interpreted as the environment where individual and group interests may be conflicting, in order to consolidate their position and access to resources. This paper, prepared on the results from a single case study and analysis of narratives, evidenced elements to allow comprehensive analysis of interpersonal relationships in the $R \& D$ area of a familiar industrial organization, in the south of the country. The analysis was constructed in comparison with the theoretical perspectives of the organizational culture, due to the relevance of the innovation management program instituted by the organization. The content of the narrative, drawn from indepth interviews with eleven technicians has provided evidence of the influence of interpersonal relationships in this area, on the criteria that guided the decisions regarding the allocation of resources and definition of the form of implementation of the research activities. It was possible to identify aspects of building interpersonal relationships, as well as the manifestation of behaviors based on the symbolic interpretation, which can contribute to the reflection on ways of management.
\end{abstract}

\section{Keywords}

Social relations, Interpersonal relationships, Organizational culture, Family business. 


\section{Introdução}

Os registros históricos, de mais diversas conotações ou tendências, têm apresentado, em profusão, relatos de conflitos que caracterizaram as relações sociais desde os primórdios da humanidade (Kluckhohn, 1963, Maior, 1967, Moraes, 2000). Também não faltam as abordagens de cunho psicológico e sociológico com o objetivo de evidenciar a ampla variedade de possíveis razões que motivaram e continuam a embasar, até os dias atuais, o estabelecimento de relações sociais, não apenas entre os indivíduos, mas, também, entre os grupos (Bunderson \& Sutcliffe, 1995, Chanlat \& Bédard, 1996). Com exceção de casos tipificados como patológicos, sob diversas caracterizações, a maioria dos autores que oferece contribuições para o tema aponta a defesa de interesses pessoais, de mais diferentes conotações, como o motivo principal (Stern, 1971, Lundin, 1977, Woodworth \& Marquis, 1977).

A organização pode ser interpretada como um recorte social, que reflete fielmente em todos os aspectos o referido universo social, multifacetado e complexo, permanentemente "em processo" e transformação, decorrente das alterações do modo de perceber e interpretar o mundo, de cada um dos indivíduos que participa ativamente da construção da referida rede social. Desta forma, se justifica o interesse dos pesquisadores em estudar o universo social com o objetivo de analisar o processo de construção das relações sociais em todos os aspectos, objetivos e subjetivos, da mesma forma como a caracterização da influência que eles podem exercer sobre a interpretação dos eventos e situações que ocorrem no âmbito social (Weick, 1995, Vaughn, 1995, Haller \& Hadler, 2006).

Uma das abordagens que se destacou no referido campo de estudo das relações sociais dentro das organizações surgiu a partir de contribuições de vertentes teóricas de Antropologia e permite caracterizar os traços idiossincráticos de cada uma das organizações como a cultura organizacional própria e diferenciada das demais organizações. Adotado e explorado, tanto em amplitude como em profundidade, por pesquisadores em Administração, Psicologia e Sociologia, o conceito de cultura organizacional ganhou terreno, corpo teórico próprio, relevância e destaque, devido à capacidade de abarcar as variáveis dos referidos campos de conhecimento, com o objetivo de explicar uma extensa variedade de fenômenos sociais (Schein, 1988, Pires \& Macedo, 2006).

Destarte a organização passou a ser interpretada como o locus de realização dos indivíduos, tanto em anseios de cunho profissional, como pessoal, por meio de construção de identidade própria e de projeção no meio social (Weick, 1995, Bunderson \& Sutcliffe, 1995). Por esse motivo é possível perceber que o tecido organizacional encontra-se permeado por interesses individuais, de diversos formatos, anseios e aspirações. Dependendo das características da cultura organizacional os referidos interesses podem ser mais evidentes e de caráter objetivo, ou serem mais subjetivos e sutis. Em muitos casos os interesses individuais podem estar até associados, em determinados graus, aos interesses de grupos sociais, desde que estes estejam alinhados, ao menos temporariamente, com os objetivos pessoais (Fischer, 1996, Haller \& Hadler, 2006).

A busca pela satisfação de seus interesses pessoais poderá levar os indivíduos ao embate por poder dentro das organizações, com base no entendimento de que o mesmo poderá facultar o acesso aos recursos e, consequentemente, à maior probabilidade em alcançar seus objetivos. No entanto, esta luta pelo poder dentro das organizações não se limita à atuação do indivíduo, sendo adotada por grupos, ou seja, por subgrupos organizacionais, aparentemente com o mesmo objetivo acesso aos recursos, reforçar a posição para obter mais poder e, finalmente, alcançar o nível de satisfação de interesses dos membros do grupo (Brass \& Burkhardt, 1993, Clegg, 1996).

Coincidentemente este comportamento não possui nenhuma característica inovadora, sendo, na sua essência, a reprodução de modelos que os relatos históricos, escritos de cunho religioso e literatura em geral, mais ou menos retocada e rebuscada, por meio de toques de romantismo, misticismo, entre outros, tem descrito, ao longo dos séculos (Maior, 1967, Moraes, 2000). Trata-se, de fato, do comportamento humano. Da mesma forma como tem sido humana a reação de todos os demais participantes do referido embate, ou seja, os oponentes, que adotam o comportamento similar, que pode variar de intensidade e, também, de formas, que se alinham com a posição hierárquica do indivíduo (e do grupo) dentro da organização e a interpretação dos limites que podem ser atingidos (Grimes, 1978; Gutierrez \& Kleiner, 1995).

A referida interpretação dos limites subsidia o processo de determinação dos objetivos individuais (do grupo) dentro do universo organizacional, da mesma forma como a concepção de estratégias a serem adotadas no embate pelo poder. Destarte o conceito (a imagem) do poder e de seus benefícios (vantagens possíveis a serem obtidas por meio deste) apresentam significativas variações de indivíduo a indivíduo, de grupo a grupo, da mesma forma como, também, a percepção da forma do embate, em termos de amplitude e limites (Stevenson \& Bartunek, 1996). 
No presente artigo realiza-se a análise compreensiva, com base no caso empírico de uma organização localizada no sul do país, das relações sociais e de conflitos dentro da área de pesquisa e desenvolvimento, com o objetivo de desenvolver uma análise compreensiva das relações interpessoais na área de $P \& D$ sob a ótica da cultura organizacional constituída e suas especificidades. Para tanto, são revisadas, na primeira etapa do trabalho, as bases teóricas concernentes ao tema, com o fito de construir as lentes necessárias para abordar o tema, de alto grau de subjetividade. A complexidade do assunto e o grau de subjetividade nortearam, também, a escolha da estratégia e do método de pesquisa, que será explicitado na seção própria - do método. A caracterização da organização segue após, juntamente com a explicitação e análise das evidências. Considerações finais e as referências encerram o presente artigo.

\section{2 o indivíduo e o Grupo - a Díade de Aproximação e Conflito}

Desde o momento de sua concepção até a morte, o ser humano vive em constante interação social. Aliás, o momento da concepção já é, per se, ao mesmo tempo, a representação simbólica e o resultado da interação social. Na continuidade, após o nascimento, no período da infância, o ser humano passa a perceber, consciente e inconscientemente, que é por meio de interação com os outros que ocorre a satisfação de suas necessidades mais primárias, como comida, abrigo, etc. Durante toda a vida é provável que o ser humano passe maior parte do tempo com outras pessoas, morando, trabalhando, se divertindo, alimentando e dormindo. Ao falecer é provável, seguindo as tradições da maioria das culturas, que seja enterrado junto aos outros de sua família, para que pudesse ser visitado periodicamente pelos vivos. Ou seja, a existência do ser humano é marcada pela forte interação social e sua sobrevivência como ser humano depende dela (Kluckhohn, 1963, Spradley, 1975, Geertz, 1989).

A filosofia também tem contribuído para o debate. Como exemplo pode ser citada a obra seminal "Suicídio" de Durkheim (1973) na qual o autor argumenta, por meio de evidências obtidas a partir de resultados decorrentes de pesquisas que ele conduziu durante vários anos, que os relacionamentos sociais protegem os indivíduos e são essenciais para a satisfação das já referidas necessidades básicas do ser humano. Ao mesmo tempo, a vertente filosófica aborda o vínculo existente entre as estruturas sociais e estruturas mentais e também entre as objetivas divisões do mundo social - em especial a divisão entre os elementos que dominam e são dominados em diferentes campos. Da mesma forma há evidências da existência de mecanismos que tendem a assegurar tanto a reprodução como a transformação das estruturas dos diferentes mundos sociais (Bourdieu, 1996).

Neste sentido o grupo pode ser conceituado como um conjunto de indivíduos que se distingue de outros grupos e de indivíduos que não pertencem ao grupo, caracterizando esta fronteira externa de formas variadas, possuindo, também, uma separação (no mínimo) intra-grupo, que visa diferenciar os líderes dos demais membros. Quando o grupo apresenta mais fronteiras internas, este passa à categoria de organização e pressupõe a existência de vários grupos internos que agem em conjunto. Estas divisões internas não são, necessariamente, divisões físicas, sendo mais de natureza psicológica, perceptiva e interpretativa (Plymire, 1993; Weick, 1995; Strati, 1998).

De forma similar Spradley (1975), Geertz (1989), Chanlat e Bédard (1996) entendem que o grupo social se constitui de coletividades de indivíduos organizados de acordo com as categorias e as regras sociais. Anônimos ou identificados por nomes, os grupos são constituídos de indivíduos de identidades similares, ou seja, com objetivos inter-relacionados, que através da construção de rede de relações sociais formam unidades mais numerosas do sistema social. Uma vez reunidos em grupo os indivíduos procuram distinguir-se dos outros indivíduos e dos outros grupos sociais adotando linguagem e símbolos próprios.

Esta base conceitual da composição e de formação dos grupos sociais também é possível aplicar para a estrutura organizacional. Trata-se, na sua essência, de um grupo de indivíduos unidos por " $n$ " diversos motivos, que podem convergir para dois principais, que são a oportunidade ou a necessidade e em formas estruturantes que variam, essencialmente, de acordo com o grau de propriedade sobre os meios de produção, a estratégia concebida, os fins organizacionais, os mercados atendidos e a estrutura de custos. Analisando esta transferência conceitual mais amiúde emerge a constatação de que o "grupo" social que constitui a referida organização apresenta a segmentação interna em vários outros grupos de indivíduos, cuja quantidade pode variar segundo as características do ambiente social (Gutierrez \& Kleiner, 1995; Stokowski, 2002).

Segundo a literatura a referida estrutura grupal pode ser organizacional, individual ou privada. A estrutura organizacional é, normalmente, formal e se reflete em regras, regulamentos, procedimentos que fomentam a constituição de papéis sociais que a organização deseja ou necessita. É com base na estrutura organizacional que o grupo constrói a sua identidade que o 
acompanha ao longo do tempo, em virtude da estabilidade que lhe é característica. Devido ao seu caráter formal ela é normalmente registrada, retratada e exposta em documentação de acesso público, como, por exemplo, em organogramas (Weber, 1976, Souza, 1978, Barnard, 1979, Vaughn, 1995, Strati, 1998).

No entanto as pesquisas de alguns autores evidenciam a importância da estrutura informal nas organizações e de como estas estruturas exercem influência na constituição da identidade organizacional dos indivíduos que constituem os grupos que formam o ambiente social da organização (Perrow, 1986, Meyer \& Rowan, 1992, Stevenson \& Bartunek, 1996, Stokowski, 2002). Pode-se afirmar que a estrutura formal representa um retrato histórico herdado das primeiras formações organizacionais, do período da Revolução Industrial, que privilegiava, naturalmente, a obediência, disciplina e hierarquia, mimetizando as estruturas das duas organizações mais tradicionais: o exército e igreja (Weber, 1976, Barnard, 1979).

Os estudos demonstram também que a estrutura individual está inscrita dentro da estrutura organizacional e evidenciam a forma pela qual os indivíduos desempenham os papéis organizacionais. Todas as pessoas dentro do grupo (da organização) desempenham os papéis dentro da estrutura formal, devidamente prescritos e referenciados, apoiando-se em seus estilos pessoais, individuais, de ser, pensar e agir. Esta estrutura, no entanto, é considerada relativa e temporária, em virtude da possibilidade de mudança de conduta, pela iniciativa do próprio indivíduo. Tanto a estrutura organizacional (formal) como a individual é observável e, portanto, pública, diferente da estrutura privada que representa a maneira pela qual cada indivíduo percebe e interpreta o grupo do qual faz parte (Souza, 1978, Clegg, 1996, Lakomski, 2001, Bernstein, 2005 ).

Vaughn (1995), Strati (1998) e Daft e Weick (2005) estudaram o interpretativismo simbólico organizacional a partir da perspectiva sociológica, que permite aos pesquisadores compreender como uma determinada organização social é percebida, vivenciada, imaginada e dirigida sob o ponto de vista de seus integrantes. O simbólico emerge das atividades e práticas dos grupos, que podem ser, desta forma, interpretadas como representações da ideologia organizacional ou de como são estabelecidas as relações com o exterior. Normalmente a construção simbólica se baseia em eventos históricos e refere-se aos indivíduos que marcaram a história da organização.

Destarte, o sujeito que ingressa no universo organizacional e passa a fazer parte de um ou mais grupos internos, em função das características do seu cargo e atribuições laborais, se defrontará com a necessidade de observar as regras de convivência não apenas da organização, como de cada um dos referidos grupos (Edwards, Belanger \& Wright, 2002). Trata-se de regras, procedimentos, normas de conduta, etc., de natureza formal e também de caráter informal, muitas vezes dicotômicas e incompatíveis entre si (Nixon, 1992). Cabe destacar que o referido conjunto de "leis" organizacionais fundamenta suas características e forma nos pressupostos visão do mundo e percepção do certo e do errado, do próprio fundador. Já as normas grupais são, normalmente, o produto decorrente da amálgama de percepções, conceitos e pressupostos dos componentes do grupo, tanto os remanescentes, como daqueles que já não fazem mais a parte do grupo, mas deixaram como seu legado a sua contribuição sobre o regramento da convivência grupal (Motta \& Caldas, 1997, Lok \& Crawford, 1999, Machado, 2005).

Como que por ironia, ao mesmo tempo em que o indivíduo sempre buscou no grupo a proteção para si e para seus próximos, uma das grandes ameaças à sobrevivência dele provém justamente de outros seres humanos, que, inclusive, podem fazer parte do próprio grupo. Trata-se de tensões naturais do indivíduo versus o grupo, o desejo de liberdade versus a necessidade de segurança do grupo. Talvez por esse motivo a sociedade, desde seus primórdios, foi compelida a estabelecer regras de convivência, com vistas à minimização dos riscos aos indivíduos que fazem parte do mesmo grupo social. Estas regras fazem parte da cultura, são compartilhadas por meio de interação social e representam a base permanente da interação. As relações sociais dentro do grupo social podem ser consideradas organizadas e sistemáticas (Selznik, 1971, Spradley, 1975) e as pessoas que integram os grupos, independente do motivo para tal (existe uma infinidade de motivos diferentes) devem se ajustar às regras do grupo (Geertz, 1989).

Para Perrow (1986) e Motta e Caldas (1997), o processo de ajuste do indivíduo também está condicionado a duas capacidades: adaptação e flexibilidade. A diferença entre adaptação e flexibilidade consiste, principalmente, na questão de preservação de princípios pessoais, decorrentes do plano de vida ou do script, que pode ser definido como a força psicológica que impulsiona a pessoa para o seu destino. O plano de vida ou script condiciona o sujeito a buscar contatos e estímulos específicos, geralmente aprendidos na infância, fazendo com que ele desenvolva a competência de lidar com os sentimentos e reagir às suas experiências de vida, determinando o uso que ele fará de suas capacidades. Enquanto que na adaptação o sujeito precede o seu ingresso no grupo com avaliação cuidadosa da forma de ser do grupo, com o objetivo de manter-se fiel aos seus 
princípios, a flexibilidade implica na necessidade do sujeito de sacrificar alguns de seus princípios constantes do script, para poder fazer parte do grupo.

Outros autores destacam que a preocupação principal do grupo organizacional, à semelhança de outros grupos sociais, deve ser a sua própria sobrevivência, que pode ser ameaçada de duas formas mais relevantes: a força destruidora externa ao grupo e a desorganização interna (entropia). Estas duas preocupações deveriam ser o foco central de todo grupo visando a sua autopreservação. Neste sentido, reconhecem-se, dentro da dinâmica grupal, três tipos de forças: (i) pressões externas, (ii) agitação interna, (iii) coesão ou força interna grupal. Normalmente os primeiros dois tipos são superados pelo grupo com base na necessidade de manter a coesão do grupo. O sucesso premia o grupo pelo fortalecimento ideológico dentro da organização (Sugato, 1994, Pires \& Macedo, 2006).

Os três fatores podem ser objeto do esforço do grupo para manter a sua identidade, mas a dinamicidade do mercado impacta inevitavelmente sobre a sua configuração e, consequentemente, autopercepção e interpretação dos eventos organizacionais. Além disso, não é possível ignorar a latência do conflito dentro do grupo. A explicação da sua existência, para Clegg (1996), Bourdieu (1998) e Lok e Crawford (1999) consiste no fato de que existe uma relação direta entre as estruturas sociais e estruturas mentais, em decorrência do embate perene e constante de determinados grupos sociais pelo poder.

Os indivíduos e os grupos sociais procuram impor as suas visões, percepções e pontos de vista, com base em seus interesses particulares, que normalmente se referem à dominação do campo, favorecendo a realização de seus objetivos. A perenização do embate se deve à necessidade do grupo dominante de preservar a sua posição e a dos grupos dominados de migrar de sua posição de dominados para a de dominação. Para tanto, ambos se utilizam de meios que possam facultar a geração de modelos mentais alinhados com seus pontos de vista, procurando consolidar o modelo social existente (grupo social que domina) ou transformá-lo (grupo social que é dominado) (Bourdieu, 1998).

A reprodução deste modus operandi percebe-se, também, no universo organizacional, onde todas as pessoas ingressam com diferenças de interesses pessoais e, ao aliar-se a determinados grupos dentro da organização, passam a compartilhar os interesses destes grupos específicos, que podem estar alinhados, ou não, com os interesses organizacionais, como também com os interesses de outros grupos organizacionais. De forma similar ao processo de dominação adotado por grupos sociais também os grupos organizacionais procuram impor seus interesses por meio de divulgação de seus particulares pontos de vista, de forma a consolidar suas posições e aumentando o poder de decisão (Grimes, 1978, Gutierrez \& Kleiner, 1995, Stevenson \& Bartunek, 1996, Bourdieu, 1998).

No entanto, o comportamento do grupo não requer apenas a adoção de decisões pertinentes e corretas, mas também a adoção de decisões alinhadas numa mesma direção por todos que fazem parte do grupo. Esta necessidade decorre do fato de que se o grupo não seguir a mesma linha mestra concebida pelo grupo e compartilhada por todos, com vistas ao alcance do mesmo objetivo, a chance de sucesso do empreendimento conjunto é mínima (Grimes, 1978, Simon, 1967, Sveiby, 1998).

\section{O viés perceptivo individual das relações sociais subjacentes à cultura organizacional}

Cada cultura particular apresenta uma forma peculiar de ver o mundo, ou seja, a perspectiva para interpretar o universo da experiência humana. Normalmente, a visão do mundo consiste de conceitos mais genéricos, abrangentes e assim, mais compreensivos sobre o funcionamento da vida. A visão do mundo auxilia, em parte, a integrar percepções e traços construídos em cima do pano de fundo cultural, além de dar o devido suporte para interpretar as regras, costumes, comportamentos e atitudes. Pelo alto grau de complexidade, abstração, subjetividade, abrangência e multiplicidade de facetas, a visão do mundo raramente consegue ser expressa com precisão (Spradley, 1975, Schein, 1988, Geertz, 1989, Schneider \& Barsoux, 2004).

Além disso, é possível constatar que a mudança, institucionalizada hoje como a característica mais relevante da história mais recente, tanto no âmbito social, como organizacional, torna o contexto atual cada vez mais complexo (Lakomski, 2001, Morin, 2002). Perenizada, institucionalizada, constante e em aceleração, a mudança representa, na sociedade de hoje o símbolo da modernidade, do desenvolvimento econômico, tecnológico e até mesmo social. Desta forma, a complexidade, para Perrow (1986) Motta e Caldas (1997), Morin (2002), Chapman e Hyland (2004), se reflete em situações e ambientes sociais, com as pessoas gravitando num universo repleto de significações. 
Desde a infância o ser humano procura decodificar os símbolos que representam o ambiente pelo qual transita, desde as palavras de seus pais e pessoas mais próximas, até suas expressões, posturas, atitudes e ações mais variadas - sempre Ihes atribuindo o sentido (Bunderson \& Sutcliffe, 1995, Blanchard, Hersey \& Johnson, 1996, Haller \& Hadler, 2006). Os sentidos, por sua vez, assumem suas próprias significações que não são universais, ficando condicionadas às linguagens que representam a cultura, ou seja, um tipo específico de código. Este código constitui um referencial que apoia a construção (ou reconstrução) da visão e do sentido do mundo percebido. O referencial é utilizado para designar, classificar, ligar e colocar ordem no universo. O referencial também define os princípios de classificação com base nos quais a sociedade pode ser segmentada em grupos distintos e fornece esquemas de interpretação que embasam o entendimento de facilidades ou de dificuldades (Vaughn, 1995, Clegg, 1996, Chanlat \& Bédard, 1996, Strati, 1998).

Com base neste entendimento é possível afirmar que o conceito de cultura é, essencialmente, semiótico, fundamentado na constatação de que o ser humano é, de certa forma, refém dos significados que ele mesmo construiu. Desta forma deduz-se que se trata de uma ciência de natureza interpretativa e não uma ciência experimental em busca de leis. A análise, portanto, consiste na escolha entre as estruturas de significação, que podem ser compreendidas como códigos estabelecidos, com o objetivo de determinar a base social e sua importância. Trata-se, muitas vezes, de enfrentar uma multiplicidade de estruturas conceituais complexas, muitas das quais se encontram sobrepostas ou amarradas umas às outras. A análise cultural é intrinsecamente incompleta e, o que é pior, quanto mais profunda, menos completa (Geertz, 1989).

No entanto a cultura ainda pode ser definida, segundo Kluckhohn (1963), como o modo de vida global de um povo, a herança social que o indivíduo adquire do seu grupo, uma forma de perceber, sentir, interpretar e acreditar, um tipo de abstração do comportamento, um conjunto de aprendizagens compartilhado. Trata-se, também, de um conjunto de orientações padronizadas para os problemas frequentes e que apresentam mesmas características, é uma forma de comportamento apreendido, um mecanismo para orientação para evidenciar atitudes e comportamento, um conjunto de técnicas para se ajustar tanto ao ambiente externo como em relação aos outros homens.

Outros autores, como Souza (1978) e Schein (1988) também percebem a cultura como um conjunto de mecanismos de controle - planos, receitas, regras, instruções - visando o domínio do comportamento. Muitos pesquisadores, como Grimes (1978), Simon (1967), Lukes (1993), Stevenson e Bartunek (1996) e Blanchard, Hersey e Johnson (1996) entendem que o ser humano pode desejar este controle (mesmo que inconscientemente) e por esse motivo ele busca o apoio em fontes simbólicas para servir de referência às suas ações no mundo (Vaughn, 1995, Strati, 1998).

Estudando o fenômeno da cultura Souza (1978) e Schein (1988) afirmam que a mesma pode ser dividida em três elementos, cada um abrangendo uma série de fenômenos interdependentes. São eles: (i) preceitos compreendidos como conjunto de normas, valores, regulamentos, política administrativa, tradições, estilos gerenciais, questões formais (leis), assim como costumes, rituais, padrões e aspectos informais que são seguidos tacitamente, (ii) tecnologia, sendo entendida como conjunto de instrumentos, processos, layout, distribuição de tarefas, divisão de trabalho e fluxo organizacional subsidiando a função técnica, metodológica científica, racional e operativa da organização, (iii) caráter como um conjunto de expressões ativas e afetivas dos indivíduos da organização, manifestações subjetivas, as percepções, os sentimentos e as reações positivas ou negativas dos sujeitos organizacionais características dos comportamentos grupais.

Para Kluckhohn (1963), Spradley (1975), Geertz (1989) as ideias, valores, atos e até mesmo as próprias emoções são produtos culturais, ou seja, produtos manufaturados com base em tendências, capacidades e disposições com as quais nascemos, mas, apesar disso, manufaturados. Com base neste entendimento é possível afirmar que também as relações sociais, sejam elas construídas espontaneamente (amizades), por laços de parentesco (núcleo familiar) ou em ambientes formalmente constituídos (organizações, vizinhança, clubes, etc) também são produtos culturais. Enfim, a cultura pode ser interpretada como um processo por meio do qual ocorre de forma permanente a construção e reconstrução da realidade - da vida.

A cultura é tratada de modo mais efetivo como um sistema simbólico, através de isolamento dos seus elementos, visando caracterizar as relações internas entre esses elementos e passando, desta forma, a especificar todo o sistema de forma geral. Neste sentido é importante focar o comportamento, pois é por meio dele, ou seja, da ação social originada no comportamento, é que as formas culturais se articulam. Estas formas encontram-se, normalmente, em várias espécies de artefatos e em vários estados de consciência. Nestes casos o significado emerge do papel desempenhado no padrão de vida decorrente (Weick, 1995, Vaughn, 1995, Strati, 1998).

Vale destacar que os sistemas culturais têm que ter um grau mínimo de coerência, pois do contrário eles não seriam sistemas. No entanto também é importante lembrar o fato de que 
narrativas de alto grau de coerência são, normalmente, inverídicas, como comprovam as ilusões de paranóicos e estórias de criminosos (Plymire, 1993, Humphreys \& Brown, 2002). Desta forma recomenda-se que o trabalho interpretativo não seja baseado apenas na rigidez da estrutura interna e ao abrigo da segurança argumentativa. Uma boa interpretação de qualquer coisa, de uma pessoa, organização ou estória, deve levar o analista ao cerne do que se propõe a analisar. A interpretação deve conseguir, preferencialmente, traçar a curva de um discurso social, fixando-o numa forma inspecionável. Neste sentido a análise cultural é (ou deveria ser) uma adivinhação dos significados e uma avaliação das conjeturas viabilizando um traçar de conclusões explanatórias (Geertz, 1989).

A importância da cultura também decorre da função que desempenha na representação do vínculo entre aquilo que os homens possuem como potencial de ser e o que eles se realmente tornam, um por um. Ser "humano" significa tornar-se individual. No entanto esta individualidade se constitui, sempre, sob a direção dos padrões culturais, sistemas de significados criados historicamente e que orientam a forma, ordem, objetivo e direção às vidas individuais. Portanto, é importante descer aos detalhes, ir além do aparente, dos tipos metafísicos e similaridades vazias, com vistas à apreensão correta do caráter individual situado contextualmente (Kluckhohn, 1963, Spradley, 1975).

A cultura organizacional, no processo de identificação organizacional representa também o elemento formador da identidade na medida em que os valores e crenças do grupo interferem na representação que este elabora acerca da organização (Weick, 1995, Strati, 1998, Symon \& Clegg, 2005). Vários autores (Demo, 2006, Bourdieu, 1998) consideram que a realidade é aquilo que o indivíduo interpreta como sendo a realidade, então existe uma grande probabilidade de que os indivíduos dentro da organização reagem ao simbolismo da figura do fundador, que permanece presente, na sua ausência. Consequentemente as características culturais podem ser consideradas também como resultado da realidade interpretada (Weick, 1995, Strati, 1998).

Por meio da aplicação empírica do seu modelo, em vinte casos, Hofstede et al. (1990) demonstraram que uma grande parte das diferenças culturais pode ser explicada por seis fatores relacionados com os conceitos já determinados da sociologia organizacional: (a) orientação operacional - a processo ou a resultados, (b) organização, que pode ser voltada a pessoas ou a tarefas, (c) ambiente de trabalho, que pode ser profissional ou patriarcal, (d) organização como sistema aberto ou fechado, ou seja, de acordo com o grau de especificidade da organização e da sua flexibilidade de interação com o ambiente onde está inserida, (e) controle organizacional e (f) abordagem, normativa ou pragmática, em relação aos fins organizacionais.

A partir de uma abordagem mais ampla do universo organizacional, quando evidencia diferenças de organização para organização, Souza (1978) aborda especificidades também entre as diversas unidades (divisões, departamentos, seções) dentro da mesma organização, formando diferentes subculturas (Bolon \& Bolon, 1994, Albert, Ashforth \& Dutton, 2000). Esta constatação das diferenças entre as unidades organizacionais incentivou vários pesquisadores a desenvolver estudos mais detalhados sobre a cultura organizacional, com destaque para iniciativas de sua mensuração.

Segundo Souza (1978), se um grupo compartilha durante algum tempo um número de experiências, lidando com problemas internos e externos, pode-se concluir que o grupo irá compartilhar uma visão de mundo. Verifica-se que os grupos com background ocupacional semelhante tendem a desenvolver culturas próprias no interior das organizações. Pessoas em associação contínua desenvolvem traços comportamentais e mecanismos culturais que são únicos àquele grupo e diferem de alguma forma dos de outros grupos e de complexo sócio-cultural mais amplo.

No entanto é importante voltar a ressaltar que a cultura organizacional nunca é uniforme e nem única. Os resultados de pesquisa para tese de doutorado de Jerry Plymire (1993), da mesma forma como os resultados de pesquisa de Bolon e Bolon (1994) corroboram a constatação de que a cultura organizacional não é representada por uma cultura única que permeia a organização como um todo. Ela é caracterizada por aspectos e traços culturais distintos cuja intensidade varia de departamento a departamento, o que sugere certa multidimensionalidade da cultura organizacional, ou seja, uma amálgama de características idiossincráticas e não apenas um composto de duas ou mais características.

\section{Método}

O método de pesquisa escolhido foi o estudo de caso, por se entender que o mesmo apresenta melhor aderência ao objetivo e às questões que nortearam o estudo. Tull e Hawkins (1976, p. 323) afirmam que "um estudo de caso refere-se a uma análise intensiva de uma situação particular". De acordo com Yin (2005), a preferência pelo uso do estudo de caso deve ocorrer em pesquisas de eventos contemporâneos, em situações em que os comportamentos relevantes não podem ser 
manipulados, mas nos quais é possível se fazer observações diretas e entrevistas sistemáticas. O estudo de caso é útil, segundo Bonoma (1985, p. 207), "... quando um fenômeno é amplo e complexo, onde o corpo de conhecimentos existente é insuficiente para permitir a proposição de questões causais e quando um fenômeno não pode ser estudado fora do contexto no qual ele naturalmente ocorre". Os objetivos do Método do Estudo de Caso não são a quantificação ou a enumeração, "[...] mas, ao invés disto: (1) descrição, (2) classificação (desenvolvimento de tipologia), (3) desenvolvimento teórico, e (4) o teste limitado da teoria. Em uma palavra, o objetivo é compreensão" (p. 206).

Desta forma, seguindo as recomendações dos autores, o estudo foi realizado com uma visão externa do pesquisador, sem envolvimento nem manipulação de quaisquer informações e, além disso, os fatos levantados pelo estudo são contemporâneos. Dentre as aplicações para o estudo de caso nesse trabalho procurou-se descrever o contexto da vida real e realizar uma avaliação descritiva. Além disso, na parte empírica deste estudo descrevem-se situações que ocorreram, confrontando-as com a teoria de forma restrita às organizações pesquisadas.

Esta descrição baseia-se na análise de narrativas, construídas a partir das entrevistas não estruturadas realizadas na organização pesquisada, com as pessoas que trabalham na área de P\&D. Após a sua transcrição, a entrevista, que, devido a seu formato, resulta, normalmente, num texto não linear e até confuso, é interpretada e reescrita na forma de um texto coeso, fluido, na primeira pessoa, em formato de narrativa. Na sequência ele é submetido à apreciação das pessoas entrevistadas, para conferência, ajustes ou contribuições. Apesar do método não ser considerado recente, ainda é tratado como inovador.

A narrativa pode ser interpretada como uma fala organizada acerca de um determinado evento, situação, assunto ou tema. Detalhando, trata-se da forma pela qual as pessoas contam suas experiências, o que elas enfatizam ou omitem, se elas se posicionam como protagonistas ou como vítimas. É importante destacar que o relacionamento que o narrador estabelece com o ouvinte faz com que a narrativa deixe de ser apenas o ato de contar algo, mas passe a ser um ato de construção de uma identidade pessoal (Riessman, 1993, Czarniawska, 2000).

De certa forma, é possível afirmar que a pesquisa por meio de narrativas escritas consiste de estudo de estórias. As estórias são onipresentes, encontradas em recortes históricos, novelas de ficção, contos, autobiografias, além de outros gêneros literários. As estórias se originam de depoimentos das pessoas sobre suas próprias histórias e sobre outras pessoas, fazendo parte de conversas cotidianas. Somados a estes depoimentos espontâneos se faz necessário que os pesquisadores de narrativas fomentem estórias orais sobre temas específicos com o objetivo de serem transcritas e analisadas. A pesquisa das narrativas é considerada como uma das abordagens de pesquisa social (Polkinghorne, 2007).

A narrativa faz parte do processo cultural no qual os sistemas simbólicos criam e são criados através do discurso e é empregada em contextos diferentes para comunicar diferentes pontos de vista. Fragmentos de narrativas maiores e versões diferentes de narrativas fazem parte do discurso cotidiano das pessoas, que são, afinal de contas, seres constituídos na linguagem baseada em significados construídos para dar sentido ao mundo em que vivem. São estes significados que fazem parte da rede de conversações, constituindo conjunto de valores, símbolos, representações, enfim, a cultura à qual acabam se integrando (Moen, 2006).

Os textos são interpretados e criados em cada palavra e frase. Por esse motivo a contextualização da narrativa torna-se obrigatória, pois o discurso é construído a partir de elementos objetivos e subjetivos que ele encontra no ambiente dentro do qual ele está inserido e os interpreta com base nos seus próprios pressupostos, formados a partir de sua própria experiência pessoal e profissional. Estes pressupostos formam filtros, padrões de comportamento, atitudes e verdades ou inverdades, que são utilizados pelo sujeito para caracterizar as determinadas situações, ou seja, servir de parâmetro de classificação da natureza da ocorrência (Riessman, 1993).

Segundo Czarniawska (2000) o método de análise de narrativas julga-se adequada para desvelar ao pesquisador os processos adotados pelo narrador para interpretar coisas, particularmente indicada para avaliar a interpretação do indivíduo de temas subjetivos, como, por exemplo, a cultura organizacional. Cabe ao pesquisador interpretar as interpretações do narrador. No entanto, é importante lembrar que o pesquisador não tem acesso direto à experiência do outro e por esse motivo o pesquisador lida e sempre vai lidar com formas diferentes e ambíguas de representação da experiência que o outro relatará por meio de fala, texto, interação e, é claro, a interpretação. Por essa razão torna-se impossível ao pesquisador manter-se neutro ou objetivo na representação da realidade. 


\section{Caracterização do Caso Estudado}

A empresa, que será tratada aqui pela denominação GAMA, é uma indústria química com quarenta e sete anos de atuação no mercado brasileiro e na América Latina, voltada ao desenvolvimento de produtos e serviços em pintura e colagem, destacando-se pela concepção de soluções inovadoras e customizadas para o setor calçadista, moveleiro, construção civil, coureiro e metal-mecânico. O seu quadro de colaboradores passa de quinhentas pessoas, alocadas nas unidades localizadas em três estados brasileiros, seu faturamento anual ultrapassou no ano passado a marca de cento e vinte milhões de dólares, baseado na comercialização de quase dois mil e quinhentos itens, entre tintas, vernizes, massas, texturas, adesivos, solventes, aditivos, entre outros.

De origem familiar, o fundador iniciou o empreendimento aos vinte e três anos de idade. Trabalhando na época, desde os treze anos de idade, como pintor de casas, decoração, letreiros e ornamentação, principalmente em bailes coloniais, o empresário sempre buscava realizar o serviço solicitado com qualidade e diferenciado, principalmente no tocante às cores utilizadas, que ele mesmo passou a elaborar. A capacidade e competência na profissão asseguraram-lhe o sucesso, clientes e independência financeira. Trabalhando como autônomo, realizado, nem pensava na possibilidade de abrir uma empresa. Mas o seu vizinho, em vias de fechamento de uma pequena fábrica de tintas procurou-lhe e ofereceu o negócio.

Com muita coragem, o jovem apostou na atividade, inovando sempre, seguindo a intuição e tendências do mercado, transformando ao longo de anos e décadas, a fabriqueta constituída de processo muito artesanal de produção, em organização moderna e tecnologicamente alinhada com as líderes do setor, nos dias atuais. Trinta e cinco anos após o início a empresa instalou a primeira filial no estado de Ceará, no ano seguinte implanta de forma pioneira o sistema do comércio eletrônico e dois anos após obtêm a certificação de qualidade pela norma internacional ISO 9001.

Depois de quarenta anos de dedicação à empresa, no ano dois mil e dois o fundador decide pelo seu afastamento da empresa, passando a se dedicar às atividades sociais, de voluntariado e lazer. Deixa a gestão aos cuidados de seus dois filhos que prosseguem na trajetória de sucesso empresarial, confirmada pela abertura de mais uma filial na região nordeste no país, desta vez no estado da Bahia, dois anos atrás. Inicia-se uma nova era para a organização, com um novo olhar sobre a forma de se relacionar com o mercado, coordenada pelo novo presidente da empresa, um dos dois filhos do fundador, administrador de empresas, convicto da necessidade de encontrar um novo foco para constituir um diferencial competitivo que pudesse distinguir a empresa de seus concorrentes de maior porte e novos entrantes. O foco escolhido é o da inovação de produtos e processos.

Na ausência de uma adesão interna às suas propostas, ele percebe a necessidade de promover uma reestruturação interna, com base na constatação de que a cultura organizacional vigente, respaldada inclusive por diretores e gerentes, criava uma expectativa de que o novo presidente se obrigaria a adaptar à organização, ao invés da organização seguir as novas determinações. $O$ processo que reestruturação, que incluiu desde a transferência de profissionais entre departamentos, matriz e filiais, promoções e até desligamentos, estendeu-se pelo período aproximado de cinco anos. Na sequência foram instituídos procedimentos, normas e regulamentos, visando introduzir o critério de meritocracia e profissionalizar as relações internas entre os colaboradores.

\section{Análise dos Resultados}

Durante o processo de pesquisa foram realizadas onze entrevistas não estruturadas, as quais foram transcritas seguindo o método de análise de narrativas, convertidas em textos narrados na primeira pessoa e submetidas à aprovação dos entrevistados. O teor das narrativas procurou contemplar tanto aspectos individuais, como profissionais, com o objetivo de avaliar a percepção e interpretação tanto de eventos objetivos como subjetivos, facultando destacar evidências que subsidiaram a construção desta seção de análise.

A maioria dos entrevistados definiu o ano 2000 como um marco na história da organização, em virtude da decisão da diretoria em buscar a diferenciação no mercado por meio de foco específico em inovação, o que, segundo o teor das narrativas, gerou uma série de conflitos internos e resistência ao novo modelo, culminando na implementação de uma série de ajustes estruturais dentro da organização, processo verificado em situações semelhantes em pesquisas conduzidas por autores como Grimes (1978), Stevenson e Bartunek (1996) e Blanchard, Hersey e Johnson (1996). Os referidos ajustes visavam criar condições necessárias para facultar a adoção da nova orientação estratégica. O termo "estrutural" refere-se, essencialmente, à modelagem organizacional, ou seja, formas de pensar, proceder, atuar e decidir, tanto em processos internos como na interação com o mercado, o que encontra alinhamento com os resultados de pesquisas acerca da realidade 
organizacional, percebida e interpretada, de Perrow (1986), Weick (1995) e Motta e Caldas (1997). Dentre eles os entrevistados, destacaram a avaliação de competências, o que resultou na constatação da presidência da empresa da necessidade de criar condições favoráveis para implementar a desejada configuração de organização inovadora.

Durante o referido processo o foco foi centrado na área de pesquisa e desenvolvimento, em virtude de sua relevante contribuição em realizar a convergência do conhecimento organizacional, pulverizado em diversas unidades organizacionais, para inovar não apenas produtos, mas também processos internos da empresa (Christensen, 2002, Cham, 2005). De uma área eminentemente técnica, designada para a execução de atividades como os testes de materiais e de novos produtos, em seus atributos mais objetivos, em sua funcionalidade, os profissionais de P\&D passaram a interagir com as unidades de marketing, produção, entre outras, para tornar o processo de desenvolvimento mais participativo/ compartilhado e aumentar, desta forma, as chances de sucesso das inovações no mercado, como já destacaram autores como Curry e Clayton (1992) e Tell (2004).

A área de $P \& D$ já contava com aproximadamente setenta profissionais, entre técnicos, assistentes e estagiários. Eram realizados ali todos os desenvolvimentos, de todos os segmentos econômicos atendidos pela organização, independente do nível de complexidade ou extensão, focando, principalmente, as alterações nas linhas atuais dos produtos. Segundo as narrativas, a forma clássica de gestão da área se caracterizava pela centralização do poder na figura do gestor, que deliberava com base em pareceres técnicos e relatórios elaborados por analistas seniores da unidade. Estes, por sua vez, contavam com o apoio de demais técnicos e estagiários para a realização de testes e produção de dados e informações demandados pelos analistas para subsidiar a elaboração dos argumentos técnicos.

A hierarquia era clara, seguindo os modelos tradicionais de administração científica e propiciava condições favoráveis para a materialização de conflitos em nível individual, de disputa pelo poder, espaço interno e autonomia decisória (Stevenson \& Bartunek, 1996). Além disso, facultava, também, o surgimento de grupos informais, conforme é comum ocorrer em organizações formais do gênero, configurando uma espécie de válvula de escape para os demais componentes da equipe, sem direito a voz ativa, buscando a sua realização como ser humano em estruturas não formais.

No entanto, o novo presidente da organização percebeu que este tipo de estrutura não favorecia a reorientação estratégica por ele pretendida, que pudesse enfatizar a criatividade, a flexibilidade, a troca de ideias, de experiências, rumo à inovação, que deveria constituir o novo diferencial competitivo da organização (Christensen, 2002, Cham, 2005). Da forma como a área de $P \& D$ estava organizada e gerenciada apenas alguns poucos "notáveis" pensavam e os demais executavam as ordens, de forma restrita, técnica e com baixíssimo nível de envolvimento nas tarefas. Se a ordem, da forma como foi repassada, não funcionava, mesmo que o técnico tivesse ideia de ajuste para fazer a adaptação necessária, não a compartilhava com o superior.

Não era, definitivamente, a imagem da organização desejada pelo novo presidente da empresa, o que o motivou a promover uma série de mudanças no departamento de P\&D. Estas mudanças foram realizadas ao longo de dois anos e compreenderam desde a transferência de técnicos para outros departamentos e migração de profissionais de outras áreas para o setor de $P \& D$ até promoções internas e, infelizmente, desligamento de pessoas que se posicionaram contra a nova orientação estratégica. Dentre eles estava o próprio gerente do setor.

O novo gestor, após alguns meses de adaptação na nova organização, propôs uma nova estrutura, que poderia, na percepção dele, reduzir a incidência de retrabalho, linhas de pesquisa imbricadas e também os conflitos de interesses que se encontravam, muitas vezes, no limiar do individual e profissional. A nova estruturação consistia em criar nove divisões, oito de pesquisa em áreas alinhadas com setores econômicos atendidos, tais como calçadista, moveleiro, construção civil, metal-mecânico, químico, entre outros, e mais uma, de aplicação, que faria a função de interface com os clientes, área de desenvolvimento e de produção. Desta forma seria possível fazer a configuração funcional da área de P\&D para atender a solicitação do presidente da empresa, ou seja, o foco em inovação, com a participação plena de todos os colaboradores, independente do nível hierárquico.

Cada uma das divisões passou a contar com um número reduzido de profissionais, que variava de oito a doze, sob a coordenação de um técnico, cuja nomeação dependia não apenas do notável conhecimento específico da divisão, mas, também, de características comportamentais de líder, que pudesse estimular o grupo a compartilhar experiências, conhecimentos e sugerir melhores soluções. Todos no grupo, dentro da divisão, independente se técnico, analista ou estagiário, deveriam ter a vez e voz no processo de pesquisa e desenvolvimento, conforme preconizado por Chapman e Hyland (2004). 
Após alguns meses, necessários para a adaptação ao novo modelo de gestão, foi possível constatar que a nova estrutura foi bem-sucedida nos atributos preconizados. As equipes se tornaram mais coesas, os coordenadores assumiram suas funções e aproximaram-se de seus colaboradores, que passaram a participar ativamente do processo de pesquisa e desenvolvimento, sugerindo e compartilhando conhecimentos. Verificou-se aceleração do ritmo de lançamentos e de soluções tecnológicas, juntamente com o aumento da taxa de assertividade e de satisfação de clientes, que corresponderam ao esforço empreendido.

No entanto, o gestor e o presidente da empresa constataram também que as divisões se isolaram e passaram a competir entre si, tanto no tocante à produtividade, refletida pelo número de desenvolvimentos com maior aceitação no mercado, como pela otimização de recursos utilizados, avaliada pela alocação de recursos financeiros, por pessoa. No decorrer do tempo esta competição entre os grupos começou a oferecer novamente os riscos que se consideravam superados, como retrabalho e duplicidade em linhas de pesquisa, em virtude de utilização de mesmas bases para o desenvolvimento de produtos comuns para algumas divisões, tais como calçadista e moveleiro.

Por esse motivo está atualmente em processo de concepção o redesenho do modelo de gestão da área, com base na proposta de integração das divisões, por meio de realização de reuniões periódicas entre os representantes divisionais, em sistema de rodízio, além de organização de palestras com profissionais externos para apresentar modelos alternativos de gestão de inovação. Os primeiros resultados sugerem que a empresa está a caminho de superar este novo obstáculo.

\section{Considerações finais}

A acirrada competição pelos mercados exige das organizações ações no sentido de ajuste de suas estratégias, suas estruturas e formas de pensar e agir. No entanto, a mudança, independente da sua amplitude ou profundidade, pode suscitar diferentes reações nas pessoas que compartilham - ambiente organizacional. Desta forma torna-se essencial a compreensão de processos sociais subjacentes à construção da rede de relações, em que os aspectos comportamentais representam o elemento catalisador, potencializando o risco de percepções e interpretações contraditórias dos indivíduos que interagem e se realizam na respectiva relação. Da mesma forma como na esfera da vida pessoal, os indivíduos e grupos podem se manifestar em defesa de seus interesses, obstaculizando a harmonização das relações sociais dentro da organização.

No caso empírico em estudo no presente artigo, foram evidenciados e analisados os comportamentos dos técnicos alocados na área de pesquisa e desenvolvimento de uma organização industrial, que passou pelo reposicionamento estratégico, com foco em inovação. Com o propósito de criar o ambiente favorável à pesquisa e concepção de soluções inovadoras, de forma a estabelecer um novo marco na história da organização e constituir um novo parâmetro para a consolidação do diferencial competitivo, o novo presidente adotou uma série de medidas que repercutiram significativamente sobre a realidade organizacional, em especial na estrutura de P\&D. Do modelo tradicional de estrutura interna do setor, que conta com aproximadamente setenta profissionais, a área foi segmentada em nove divisões, alinhadas com os setores econômicos atendidos, procurando, desta forma, reduzir os índices de retrabalho, linhas de pesquisa imbricadas, estimular o compartilhamento de conhecimentos e de experiências, alavancando a inovação de produtos.

O projeto foi considerado bem-sucedido, tanto na dimensão técnica e visível, objetivada com base em evidências de conclusão de projetos de pesquisa em atraso, lançamento de novos estudos, implementação de soluções customizadas, como no nível de coordenação, em que a maior proximidade do líder se refletiu na maior integração da equipe dentro de cada segmento, interagindo e cooperando. No entanto, a estrutura de gestão, fundamentada na organização divisional, na qual cada uma das novas unidades recebeu suas próprias metas, orçamento e autonomia para gerenciar linhas de produtos e com avaliação por indicadores de desempenho por grupo, foi novamente questionada, por suscitar diferentes interpretações pelos integrantes dos grupos e, principalmente, de seus coordenadores.

Ao mesmo tempo em que o acirramento de competição entre os grupos constituídos facultou a consolidação do modelo favorável para a inovação, tanto de produtos como de processos, ressurgiram as dificuldades de relacionamento interpessoal, desta vez entre os grupos. $O$ compartilhamento dos resultados das pesquisas foi obstaculizado, implicando a condução de estudos em duplicidade, retrabalho, desperdício de tempo e recursos aplicados. A competição entre os grupos de pesquisa exigiu da diretoria reflexão sobre seu próprio posicionamento em relação à forma de lidar com questões de relacionamento interpessoal, de natureza mais subjetiva e sutil.

Atualmente uma nova proposta está sendo estudada, com o objetivo de ajustar os processos e otimizar a utilização de recursos internos, visando harmonizar o ambiente interno, reduzir os níveis 
de competição, sem, no entanto, abandonar o objetivo de fomentar a consolidação do modelo de gestão baseado na inovação. O novo presidente e sua diretoria descobriram no modelo estereotipado da área técnica uma complexidade ímpar, com realidade percebida e interpretada pelos atores organizacionais, contexto que não permite adoção de medidas simples e superficiais, mas que, ao mesmo tempo, pode proporcionar novas oportunidades na construção de diferenciais competitivos. Acredita-se que a análise da situação empírica pode propiciar condições de reflexão acerca do processo de gestão de pessoas nas áreas técnicas.

\section{Referências}

Albert, S., Ashforth, B. E, \& Dutton, J. E. (2000) Organizational identity and identification: charting new waters and building new bridges. Academy of Management Review, 25(1), 13-17.

Barnard, C. I. (1979) As funções do executivo. São Paulo: Atlas.

Bernstein, M. (2005) Identity Politics. Annual Review of Sociology, 31(1), 47-74.

Blanchard, K H., Hersey, P., \& Johnson, D. (1996). Management of Organizational Behavior: Utilizing Human Resources. Englewood Cliffs: Prentice Hall.

Bolon, D. S, \& Bolon, D. S. (1994). A reconceptualization and analysis of organizational culture. Journal of Managerial Psychology, 9(5), 22-27.

Bonoma, T. V. (1985) Case research in marketing: opportunities, problems, and process. Journal of Marketing Research, 22(2), 199-208.

Bourdieu, P. (1998) O poder simbólico. Rio de Janeiro: Bertrand Brasil.

Bourdieu, P. (1996) The state nobility. Stanford: Stanford University Press.

Brass, D. J., \& Burkhardt, M. E. (1993). Potential power and power use: An investigation of structure and behavior. Academy of Management Journal, 36(3), 441-470.

Bunderson, S. J., Sutcliffe, K. M. (1995) Work History and selective perception: Fine-tuning what we know. Academy of Management Best Papers Proceedings, 459-463.

Chanlat, A., Bédard, R. (1996). Palavras: a ferramenta do executivo. In: Chanlat, J-F. (Org.). O indivíduo na organização: dimensões esquecidas (pp. 125-148) (3 ed.). São Paulo: Atlas.

Cham, K. W. (2005) A estratégia do Oceano Azul - como criar novos mercados e tornar a concorrência irrelevante ( 5 ed.). Rio de Janeiro: Elsevier.

Chapman, R., \& Hyland, P. (2004). Complexity and learning behaviors in product innovation. Technovation, 24(7), 553-561.

Christensen, J. F. (2002). Corporate strategy and the management of innovation and technology. Industrial and Corporate Change, 11(2), 263-288.

Clegg, S. (1996). Poder, linguagem e ação nas organizações. In: Chanlat, J-F. (Org.). O indivíduo na organização: dimensões esquecidas (pp. 47-66) (3 ed.). São Paulo: Atlas.

Curry, S. J., \& Clayton, R. H. (1992). Business Innovation Strategies. Business Quarterly, 56(3), 121116.

Czarniawska, B. (2001). Anthropology and Organizational Learning. In Meinolf Dierkes, Ariance B. Antal, John Child, \& Ikujiro Nonaka. Handbook of Organizational Learning and Knowledge (pp. 118136). New York: Oxford University Press.

Daft, R., \& Weick, K. E. (2005) Por um modelo de organização concebido como sistema interpretativo. Revista de Administração de Empresas, 45(4), 73-86.

Demo, P. (2006) Pesquisa e Informação Qualitativa. São Paulo: Papirus.

Durkheim, É. (1973). O suicídio: estudo de sociologia. Lisboa: Presença.

Edwards, P., Belanger, J., \& Wright, M. (2002). The social relations of productivity: A longitudinal and comparative study of Aluminium Smelters, Relations Industrielles, 57(2), 309-330.

Fischer, G.-N. (1996). Espaço, identidade e organização. In Chanlat, J-F. (Org.). O indivíduo na organização: dimensões esquecidas ( 3 ed.). São Paulo: Atlas.

Geertz, C. (1989). A interpretação das culturas. Rio de Janeiro: LTC.

Grimes, A J. (1978). Authority, power, influence and social control: A theoretical synthesis. Academy of Management Review, 3(4), 724-735. 
Gutierrez, K. A., \& Kleiner, B. H. (1995). Enhancing personal power in organizations. Agency Sales, $25,52$.

Haller, M., \& Hadler, M. (2006). How social relations and structures can produce happiness and unhappiness: An international comparative analysis. Social indicators research, 75(2), 169-216.

Hofstede, G., Neuijen, B., Ohayv, D. D., \& Sanders, G. (1990). Measuring organizational cultures: A qualitative and quantitative study across twenty cases. Administrative Science Quarterly, 35(2), $286-$ 316.

Humphreys, M., \& Brown, A. (2002) Narratives of organizational identity and identification: a case study of hegemony and resistance. Organizational Studies, 23(3), 421-447.

Kluckhohn, C. (1963). Antropologia: Um espelho para o homem. Belo Horizonte: Itatiaia.

Lakomski, G. (2001). Organizational change, leadership and learning: culture as cognitive process. International Journal of Educational Management, 15(2), 68-77.

Lok, P., \& Crawford, J. (1999). The relationship between commitment and organizational culture, subculture, leadership style and job satisfaction in organizational change and development. Leadership \& Organization Development Journal, 20(7), 365-374.

Lukes, S. (1993). Power: a radical view. New York: MacMillan.

Lundin, R. W. (1977). Personalidade: uma análise do comportamento. São Paulo: Editora Pedagógica e Universitária Ltda.

Machado, H. V. (2005). Identidade organizacional: um estudo de caso no contexto da cultura brasileira. RAE eletrônica, 4(1).

Maior, A. S. (1967). História Geral. São Paulo, Editora São Paulo.

Meyer, J. W., \& Rowan, B. (1992) Institutionalized organizations: formal structures as myth and ceremony. In John W. Meyer, \& Richard W. Scott. Organizational Environments: ritual and rationality (pp. 261-282). Updated Edition. London: Sage.

Moen, T. (2006). Reflections on the narrative research approach. International Journal of Qualitative Methods, 5(4), 56-69.

Moraes, J. G. V. de. (2000). Caminhos das Civilizações. São Paulo: Saraiva.

Morin, E. (2002). As Duas globalizações: complexidade e comunicação, uma pedagogia do presente (2 ed.). Porto Alegre: Sulina Universitária, EDIPUCRS.

Motta, F. C., \& Caldas, M. (1997). Cultura organizacional e cultura brasileira. São Paulo: Atlas.

Nixon, B. (1992). Developing a new culture for organisations in the 90's. Management Education and Development, 23(1), 33.

Perrow, C. (1986). Complex organizations: a critical essay. New York: McGraw-Hill.

Souza Pires, J. C. de, \& Macêdo, K. B. (2006). Cultura organizacional em organizações públicas no Brasil. Revista de Administração Pública, 40(1), 81-105.

Plymire, J. (1993). The Depth Psychological Implications of Organizational Culture. Tese de Doutorado da Pacifica Graduate Institute.

Polkinghorne, D. E. (2007). Validity issues in narrative research. Qualitative inquiry, 13(4), 471-486.

Riessman, C. K. (1993). Narrative analysis. London: SAGE.

Schein, E. H. (1988). Organization Culture and Leadership. San Francisco: Jossey Bass.

Schneider, S., \& Barsoux, J. (2004). Culture and organization. In C. A. Barlett, S. Goshal, \& J. Birkinshaw. Transnational Management: text, cases and readings in cross-border management (4 ed.). New York: McGraw-Hill.

Selznik, P. (1971). A Liderança na Administração: uma Interpretação Sociológica. Rio de Janeiro: Fundação Getúlio Vargas.

Simon, H. A. (1991). Bounded rationality and organizational learning. Organization Science, 2(1), 125-134.

Souza, E.L.P. (1978). Clima e Cultura organizacional: como se manifestam e como se manejam. São Paulo: Edgard Blucher.

Spradley, J. P. (1975). Anthropology: The cultural perspective. New York: John Wiley \& Sons. 
Stern, W. (1971). Psicologia Geral. Lisboa: Fundação Calouste Gulbenkian.

Stevenson, W. B., \& Bartunek, J. M. (1996). Power, interaction, position, and the generation of cultural agreement in organizations. Human Relations, 49(1), 75-104.

Stokowski, P. A. (2002). Languages of place and discourses of power: Constructing new senses of place. Journal of Leisure Research, 34(4), 368.

Strati, A. (1998). Organizational symbolism as a social construction: A perspective from the sociology of knowledge. Human Relations, 51(11), 1379-1402.

Lahiry, S. (1994). Building commitment through organizational culture. Training \& Development, 48(4), 50-53.

Sveiby, K. E. (1998). A nova riqueza das organizações. Rio de Janeiro: Campus.

Symon, G., \& Clegg, C. (2005). Constructing identity and participation during technological change. Human Relations, 58(9), 1141-1166.

Tell, F. (2004). What do organizations know? Dynamics of justification contexts in R\&D activities. Organization, 11(4), 443-471.

Tull, D. S., \& Hawkins, D. I. (1976) Marketing Research, Meaning, Measurement and Method. London: Macmillan.

Vaughn, M. A. (1995). Organization Symbols: An Analysis of their types and functions in a reborn organization. Management Communication Quarterly, 9(2), 219-250.

Weber, M. (1976). Os Fundamentos da Organização Burocrática: uma construção do tipo ideal. In E. Campos (Org). Sociologia da Burocracia (pp. 16-27). Rio de Janeiro: Zahar.

Weick, K. E. (1995) Sensemaking in organization. London: Sage.

Woodworth, R. S., \& Marquis, D. G. (1977) Psicologia. São Paulo: Companhia Editora Nacional.

Yin, R. (2005) Estudo de caso: planejamento e métodos. Porto Alegre: Bookman. 